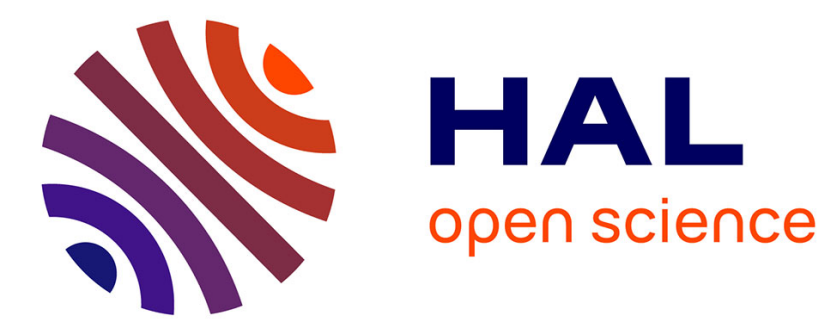

\title{
La nation retrouvée. De la RDA à l'Allemagne
}

\author{
Anne-Marie Le Gloannec
}

\section{To cite this version:}

Anne-Marie Le Gloannec. La nation retrouvée. De la RDA à l'Allemagne. Politique étrangère, 1990, 55 (1), pp.45-52. 10.3406/polit.1990.3916 . hal-01009600

\section{HAL Id: hal-01009600 \\ https: / hal-sciencespo.archives-ouvertes.fr/hal-01009600}

Submitted on 18 Jun 2014

HAL is a multi-disciplinary open access archive for the deposit and dissemination of scientific research documents, whether they are published or not. The documents may come from teaching and research institutions in France or abroad, or from public or private research centers.
L'archive ouverte pluridisciplinaire HAL, est destinée au dépôt et à la diffusion de documents scientifiques de niveau recherche, publiés ou non, émanant des établissements d'enseignement et de recherche français ou étrangers, des laboratoires publics ou privés. 


\title{
Le Gloannec
}

\section{La nation retrouvée. De la RDA à l'Allemagne}

In: Politique étrangère Nº1 - 1990 - 55e année pp. 45-52.

\begin{abstract}
The Nation Lost and Found. From GDR to Germany, by Anne-Marie Le Gloannec

The inter-German time upsets diplomatie calendars. The GDR exists no more because it never existed : there was no free and independent state but interpenetration of two societies or rather an unequal exchange. Though the influence of the FRG in the GDR was stronger, West German society did not remain entirely without some Eastern influence. Therefore the reunified Germany will not be merely a big Federal Republic. Though the fall of communist regimes and the reunification of the two Germanies bring more security to Germany and Europe, they also lead Bonn and West Berlin — and later Berlin — into economie and political turbulence. Will Germany be able to stand by its pledges to the outside world?
\end{abstract}

\section{Résumé}

Le temps interallemand bouscule les calendriers diplomatiques. C'est que la RDA n'existe plus parce qu'elle n'a jamais existé : il n'y eut jamais d'Etat libre et indépendant, au contraire une interpénétration de deux sociétés ou plutôt un échange inégal. Si l'influence de la RFA en RDA fut prépondérante, la société ouest-allemande n'échappa pas entièrement à certaines influences orientales. L'Allemagne réunifiée ne sera donc pas simplement une grande République fédérale. Si la chute des régimes communistes et la réunification des Allemagnes apportent à l'Allemagne et à l'Europe plus de souveraineté mais non nécessairement plus de sécurité, elles entraînent aussi Bonn et Berlin-Est - et plus tard Berlin - dans des turbulences économiques et politiques. L'Allemagne parviendra-t-elle à tenir ses engagements vis-à-vis de l'étranger ?

Citer ce document / Cite this document :

Le Gloannec. La nation retrouvée. De la RDA à l'Allemagne. In: Politique étrangère Nº1 - 1990 - 55e année pp. 45-52.

doi : 10.3406/polit.1990.3916

http://www.persee.fr/web/revues/home/prescript/article/polit_0032-342X_1990_num_55_1_3916 


\section{\begin{tabular}{l|l} 
Anne-Marie LE GLOANNEC * & $\begin{array}{l}\text { La nation retrouvée. } \\
\text { De la RDA à l'Allemagne }\end{array}$
\end{tabular}}

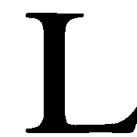

e 18 mars 1990, les premières élections libres et démocratiques se dérouleront en RDA. Ce seront aussi les dernières. Les Allemands s'entendront alors pour constituer un seul espace économique et monétaire - dès avril peut-être - voire étatique - avant la fin de l'année. Le temps interallemand bouscule les calendriers diplomatiques, à Washington et à Moscou, à Londres et à Paris ou encore à Bruxelles, au siège de la Communauté européenne et à celui de l'OTAN. C'est que la RDA n'existe plus. Chaque jour, 2000 à 3000 Allemands de l'Est gagnent l'Ouest et les intentions de départ ne font que croître : $62 \%$ de la population seulement veut rester à tout prix au pays contre $84 \%$ en novembre $1989^{\prime}$. Le tissu économique et le tissu social se délitent, l'un et l'autre fragiles, le premier parce que quarante-cinq ans d'erreurs et de négligence l'abîmèrent, le second parce qu'il n'y eut jamais véritablement une société intégrée, tout au plus des poches communautaires. Autrement dit, quelle que soit l'ampleur de la faillite économique que certains, à l'Est plus qu'à l'Ouest, à gauche plus qu'à droite, contestent par crainte de "brader» l'Allemagne orientale à l'acheteur ouest-allemand, la RDA n'existe plus parce qu'elle n'a jamais existé : il n'y eut jamais d'Etat libre et indépendant. Au contraire, il y eut toujours, même partielle et parfois déclinante, une interpénétration des deux sociétés ou, plus exactement, un échange inégal tel que la moins intégrée, la moins structurée, la moins démocratique et la moins riche des deux sociétés dépendit de la plus libérale. Si l'influence, voire la toute-présence, de la République fédérale en République démocratique fut prépondérante, la société ouest-allemande n'échappa pas toutefois entièrement à certaines influences orientales, ainsi dans les années 70 et 80 où une partie des Allemands de l'Ouest crurent pouvoir trouver à l'Est trace de leur identité. L'Allemagne réunifiée ne sera donc pas purement et simplement une grande République fédérale. Les Allemands de l'Est y laisseront leur empreinte et ceux de l'Ouest s'en trouveront transformés. L'unification des Allemagnes aura, pour les Alliances et pour l'Europe, des conséquences qui ne se résumeront pas à une seule modification des champs de force internationaux.

\footnotetext{
* Chargée de recherches au Centre d'études et de recherches internationales (CERI) de la Fondation nationale des sciences politiques, Paris.

1. Sondage est-allemand cité par David Goodhart, « East Germans Learn to Live with Language of Collapse », The Financial Times, 12 février 1989.
} 


\section{Un Etat factice}

Même si les manifestants de Leipzig attendirent les premières semaines de novembre pour réclamer l'unification des Allemagnes, on pouvait néanmoins penser qu'une formidable dynamique interallemande s'enclencherait, ne serait-ce que parce que les liens interallemands ne se brisèrent jamais. Les liens familiaux - qui renvoient une image à plat, une sorte d'instantané des relations interallemandes - ne se sont jamais vraiment distendus ou encore, distendus, ils se sont reconstitués : l'émigration d'Est en Ouest s'était certes tarie entre 1961 et 1984 - c'est-à-dire de la construction du Mur à l'entrouverture des frontières qu'Erich Honecker, premier secrétaire du SED, autorisa, par libéralité plus que par libéralisme - mais elle reprit après 1984. Toujours est-il qu'avant les départs massifs de 1989 et 1990, $19 \%$ des Allemands de l'Ouest avaient des parents de l'autre côté de l'Elbe et $31 \%$ - c'est-à-dire plus de 18 millions d'Allemands de l'Ouest avaient des parents et amis dans une RDA de 16,5 millions d'habitants. $32 \%$ des Allemands de l'Ouest se rendaient en RDA. On peut supposer qu'il s'agissait plus ou moins des mêmes individus : on allait en RDA pour voir les siens.

C'est ainsi que, de 1972 à 1985, on dénombra plus de 82 millions de passages d'Ouest en Est, un peu comme si les 18 millions d'Allemands de l'Ouest qui ont parents et amis en RDA leur avaient rendu visite quatre fois en quinze ans. Ce furent autant de voitures qui sillonnèrent les routes de l'Elbe à l'Oder, de la Thuringe à la Baltique, autant de vêtement vus, d'objets montrés ou donnés, de comportements analysés ou d'informations échangées. On a eu tort, à cet égard, de comparer tout bonnement ces voyages en RDA à ceux des quelque 5 millions de touristes ouest-allemands qui, chaque année, se rendent en Autriche. Les Autrichiens ont un niveau de vie sinon égal à celui des Allemands de l'Ouest, du moins comparable, et ils sont libres. Avec les Allemands de l'Ouest, c'est un peu de leur Allemagne qui vint à ceux de l'Est. Des nouvelles télévisées aux objets de consommation, de la mode aux modes, des normes industrielles à celles de comportement, des cadeaux personnels aux subsides publics, la société estallemande vécut et vit encore par l'ouest-allemande - même si les emprunts ne furent pas de simples copies.

Mais, tout autant que la société, le régime et l'Etat est-allemands n'ont cessé de dépendre de la République fédérale, sur les plans économique et politique. Qu'il se soit agi de soutenir l'économie est-allemande ${ }^{2}$ ou de stabiliser le gouvernement de Berlin-Est, l'essence de la Deutschlandpolitik, conçue par Egon Bahr et mise en pratique par les divers gouvernements fédéraux, consista justement à se rapprocher du SED jusqu'à parfois ignorer l'opposition, pour que, rassuré, celui-ci entame des réformes. Cette politique échoua et réussit tout à la fois. Elle servit le régime est-allemand dans la mesure où les concessions ouest-allemandes ne s'accompagnaient pas toujours de contreparties est-allemandes - au grand regret des critiques de la politique fédérale. Les dirigeants de Berlin-Est surent également tirer parti de la proximité ou de la toute-présence de la République fédérale pour vider de temps à autre l'abcès de la dissidence en envoyant les

2. On estime que subventions et subsides, publics et privés, furent de 4 milliards de marks allemands - 2 milliards de la main publique et 2 milliards du ressort privé. 
trublions à l'Ouest. La télévision ouest-allemande elle-même put avoir un effet stabilisateur, offrant une soupape aux mécontents qui préféraient le rêve à l'action.

Au bout du compte toutefois, cette dépendance, comme toute situation de ce type, s'avéra déstabilisatrice. La société et le gouvernement ouestallemands ne compensèrent pas les faillites du régime, ils les révélèrent au contraire et nourrirent aussi les foyers de contestation. Ils agirent ainsi doublement, fournissant des appuis, matériels (subventions...), intellectuels (livres) et psychologiques, à l'opposition est-allemande et, indirectement, en démontrant que les fondements du régime étaient pure rhétorique. Les dirigeants est-allemands avaient prétendu créer un Etat et un homme nouveaux, socialistes : le contraire était vrai. La société et l'Etat estallemands dépendaient de la société et de l'Etat ouest-allemands - ils en dépendaient d'ailleurs d'autant plus qu'ils ne dépendaient que d'eux. $\mathrm{Ne}$ pouvant aller à l'Ouest, les Allemands de l'Est firent venir à eux la République fédérale : ils n'eurent ainsi accès qu'à un seul monde, celui de la République fédérale, qui leur apporta sa version du monde. La RDA ne cessa donc d'être une société pénétrée. Parce que ses dirigeants la voulaient fermée, elle fut pénétrée. Parce qu'ils la voulurent autarcique, elle fut dépendante. Telle est la dialectique de la fermeture et de l'ouverture.

A partir de ce constat, il n'y eut pas d'autre politique possible que celle de l'ouverture totale à la République fédérale, de la dépendance reconnue, donc, quasiment, de la réunification. L'entrouverture des frontières de 1984 à 1989 démontra l'ampleur du dilemme : plus le régime entrouvrait les frontières, plus il suscitait de ressentiments, créant une société à deux vitesses car il autorisait à voyager ceux qui avaient parents et amis en République fédérale mais non les autres. La fermeture et l'entrouverture impossibles, il ne restait plus qu'à ouvrir les frontières - et à laisser libre cours aux flux interallemands.

Est-ce à dire que l'option d'une troisième voie n'a jamais existé ? Il y eut en germe une culture politique est-allemande qui se développa à l'abri du Mur, de 1961 à 1989. Dès les années 60, des observateurs, d'abord étrangers puis allemands, crurent, à raison, reconnaître des traces d'une identité est-allemande qui se traduisit par une certaine fierté, pour les uns, d'avoir lutté contre le «fascisme allemand »et, pour les autres, d'avoir reconstruit cette partie d'Allemagne malgré l'adversité. Cette culture politique puisait, pour simplifier, à deux sources : d'une part, aux sources protestantes et sociales-démocrates, culture faite d'austérité et de rigueur et qu'Oswald Spengler décrit, certes avec partialité mais non sans finesse ${ }^{3}$. Les intellectuels incarnent notamment cette culture. Ils ont souhaité, ces derniers mois, l'avènement d'un libéralisme politique plus qu'économique qui leur permettrait de rejeter le capitalisme par peur de ses dégâts et de refuser le compromis politique, par peur des compromissions. En un mot - que forgea Volker Braun -, ils veulent fuir l'"opportunisme de la liberté " ${ }^{4}$.

L'autre ferment d'identité est-allemande est à la fois plus ancien et plus récent, identité de la Heimat, du repli sur le pays, sur le connu, le pré-

3. Oswald Spengler, Socialisme et prussianité paru en traduction française.

4. Article paru dans Neues Deutschland, 8 décembre 1989. 
moderne, identité que la fermeture des frontières accentua - identité d'enfermement. Avec la construction du Mur, les Allemands de l'Est se retrouvèrent entre eux, dans un Etat à leur image, ethniquement et socialement homogène puisque le Mur s'était refermé sur ces Allemands qui n'avaient pas quitté le pays, c'est-à-dire la petite bourgeoisie et la classe ouvrière ! Etat de petites gens, la RDA offrait, d'une certaine façon, un cadre rassurant, loin du " grand monde " - dans tous les sens du terme loin des complexités de la modernisation ${ }^{5}$.

Cette identité pré-moderne rejoint celle de l'intelligentsia, à la fois pré et post-moderne, l'une et l'autre renouant avec des traditions allemandes, antioccidentales, celle de la Kultur allemande, des «vraies valeurs », par opposition à la Zivilisation occidentale, technicienne. Mais l'ouverture du Mur menaça très vite l'une et l'autre. D'une part, l'ouverture, la modernisation économique et intellectuelle les balayeront. Il ne peut y avoir en effet modernisation économique sans modernisation politique et le libéralisme s'accompagnera nécessairement des vices qui lui sont propres. Ouvriers et bourgeois ont déjà choisi de rallier l'Occident pour le meilleur et pour le pire, délaissant ces intellectuels qui, le 29 novembre 1989, lancèrent un « appel pour le pays", en se discréditant ${ }^{6}$. D'autre part, y a-t-il une identité est-allemande qui ne soit pas aussi allemande, entretenue notamment par les liens de dépendance décrits plus haut ? De part et d'autre de l'Elbe se dessinent des affinités croisées, protestante, sociale-démocrate ou encore conservatrice, que traduisent les rapprochements entre partis politiques des deux Allemagnes?

\section{Le droit à l'émotion}

Si la RDA n'a jamais pénétré la société ouest-allemande, les interactions entre République fédérale et République démocratique n'en ont pas moins influencé la société et l'Etat ouest-allemands dans la mesure où elles renvoyaient à l'inconnue, à la nation. Dès l'origine en effet, l'Etat ouestallemand reposa sur deux piliers fondateurs, théoriquement conciliables mais en fait antithétiques puisque la partition de l'Allemagne s'avérait durable. D'un côté, la République fédérale s'ouvrit à l'Occident et à ses valeurs, tournant le dos à l'Est et au fait national, "société avant d'être Etat " ${ }^{8}$, Etat à peine doté d'attributs symboliques, sans fête commémorative puisque le 17 juin, date à laquelle fut écrasée la révolte ouvrière est-allemande, ne fondait pas l'Allemagne et encore moins la République fédérale, mais symbolisait au contraire ce qui devait être un Etat transitoire. De l'autre côté, la République fédérale revendiqua non l'héritage du Reich mais la continuité d'avec le Reich, incarnation du Reich qui, légalement, n'a jamais cessé d'exister. Tendue entre deux pôles, celui de l'unité nationale et celui

5. Voir Günter Gaus, Wo Deutschland liegt : eine Ortsbestimmung, Hoffmann und Campe, Hambourg, 1983.

6. Texte reproduit dans $T A Z, 29$ novembre 1989.

7. Ces pages s'appuient notamment sur Anne-Marie Le Gloannec, La Nation orpheline. Les Allemagnes en Europe, Calmann-Lévy, Paris, 1989, notamment deuxième partie, ainsi que, du même auteur, "RDA : des Allemands pas comme les autres ", L'Histoire, février 1990 et "la RDA survivra-t-elle en $1990 \approx$ ?, Politique internationale, $\mathrm{n}^{\circ} 46$, hiver 1989-1990, pp. 70-79.

8. Selon la formule d'Ernst Nolte, à l'époque où il était encore un historien respectable, dans Deutschland und der kalte Krieg, Klett-Cotta, Stuttgart, 1974. 
de l'unification européenne, la République fédérale voulut concilier l'un et l'autre jusqu'à ce que le traité de 1972 bouleverse la donne. Par ce traité, chaque Etat reconnut l'autre de sorte que chacun dut redéfinir, d'une façon ou d'une autre, les fondements sur lesquels il s'était constitué. En d'autres termes, dès lors que la confrontation idéologique et politique entre les deux Etats marquait le pas, il importait de redéfinir la filiation identitaire entre Etats et nation.

Dès le début des années 70 , se dessina en République fédérale une quête d'identité d'abord par le bas puis d'en haut: redécouverte du folklore, de l'histoire locale, du terroir, de l'environnement, des pays allemands et querelle des historiens sont liées par un même fil, celui de la quête identitaire, un peu comme si les Allemands recherchaient alors ce qu'ils avaient perdu depuis plus que d'autres, un peu comme s'ils avaient voulu se réapproprier le droit aux sentiments et le droit au passé. Lothar Baier décrivit avec finesse et ironie cette redécouverte des racines: alors que l'émigration courait encore dans les têtes, "dans l'ombre (...) une curieuse petite plante commença de fleurir, sans être remarquée. La vie, en haut, dans ce pays, continuait de s'enfuir alors qu'en bas, dans la pénombre chaude et humide, elle avait pris racine. Un amour du pays, curieux, honteux, quelque peu rougissant, avait commencé à grandir, déguisé en deuil - deuil de la destruction d'idylles dignes d'être préservées " 9 . Dès la fin des années 70 , cette quête devait entraîner le SED à vouloir lui aussi récupérer l'histoire, histoire inscrite dans un territoire qu'il croyait est-allemand, celui de la Prusse et du luthéranisme. Cette histoire-là conduisit néanmoins à l'histoire allemande et à un présent interallemand : on le vit en 1983 lorsqu'Allemands de l'Ouest et de l'Est se pressèrent côte à côte, à Eisleben et à Wittenberg, pour célébrer conjointement le luthéranisme.

L'Ouest redécouvrit ainsi le passé et le présent, le passé allemand, en RDA notamment, qui le conservait à l'abri de la modernisation. En redécouvrant le passé allemand en RDA, la République fédérale redécouvrit aussi le présent est-allemand. Après avoir disparu des consciences ouest-allemandes dans les années 60 et 70 , la RDA revint subrepticement, sous forme d'images - puis massivement sous forme d'émigrés. Ceux qui se rendirent alors en RDA en rapportèrent des images de nostalgie : " $L a R D A$, un musée en plein air qui respire la nostalgie », note Günter Gaus, le premier représentant de République fédérale à Berlin-Est, et Manfred Ackermann, qui lui aussi travailla à la représentation permanente, voulut voir dans la RDA une «Allemagne à l'état pur » (Deutschland in Reinkultur).

Certains Allemands avaient cru enterrer la nation, elle se reconstitua, à l'ombre des rencontres des années 70 et surtout 80 , elle crût, elle s'exposa le 9 novembre 1989. Certains avaient voulu prétendre au postnational, mais ils avaient parlé pour une minorité. On ne s'étonnera pas si, le 9 novembre, les Allemands ont laissé exploser des sentiments de joie interallemande joie des retrouvailles avec l'autre et avec soi-même, droit à l'émotion, légitimé par la libération de l'Europe de l'Est et de l'Allemagne orientale. La chute du Mur levait les derniers tabous, le voile du non-dit national. En filigrane s'inscrivait la levée des contraintes de la dernière guerre : la fin du

9. Lothar Baier, «Bewegte BRD », dans Hans-Jürgen Heinrichs, ed., Abschiedsbriefe an Deutschland, Qumran, Francfort, pp. 21-22. 
statut quadripartite à Berlin, la fin de l'antagonisme idéologique et donc militaire entre l'Est et l'Ouest.

\section{Les coûts de la recomposition}

Les retrouvailles interallemandes revêtent donc des implications politiques considérables. Naguère la présence de l'autre Allemagne avait déjà pesé sur la politique fédérale. Dans la mesure en effet où les deux Etats naquirent d'une nation divisée, fruits d'interventions étrangères, points d'ancrage d'alliances idéologico-militaires aux raisons opposées, les deux Etats souffrirent de dilemmes, tiraillés entre raison d'Etat et raison de nation, entre sécurité et souveraineté. A mesure que la confrontation Est-Ouest s'apaisait, que l'anticommunisme s'effaçait des esprits et des cœurs, les hommes politiques ouest-allemands voulurent utiliser les relations avec l'autre Allemagne, sinon pour relâcher un peu leurs liens avec l'Ouest, du moins pour démultiplier les contraintes en les multipliant.

Dans chacune des trois dimensions de la politique étrangère de Bonn relations avec les Etats-Unis, relations avec l'Europe orientale et Ostpolitik - la République fédérale était entravée par des contraintes multiples, d'ordre juridique, psychologique et politique. Dans la quatrième dimension, les Allemands de l'Ouest furent sinon plus libres, du moins en terrain connu, partageant avec d'autres Allemands des contraintes en partie analogues. Au demeurant, plus il y avait de dimensions à la politique étrangère, aussi contraignantes fussent-elles, plus il était possible de jouer entre les unes et les autres, des unes aux autres, plus il était facile en somme d'introduire du jeu entre les différentes sphères et d'accroître la marge de manœuvre du gouvernement fédéral.

Avec la fin du communisme et la fin de l'antagonisme Est-Ouest, avec la réunification des Europes et des Allemagnes, Bonn, Berlin-Est ou Berlin, prochaine capitale de l'Allemagne réunifiée, ne devront plus importer leur sécurité au prix de la souveraineté du pays. C'est là l'une des principales conséquences du rapprochement et de l'unification des Allemagnes. Que l'Allemagne soit ou non neutralisée ou qu'elle s'intègre à l'OTAN, elle deviendra souveraine. C'est précisément pour cette raison qu'il est préférable de l'encadrer dans un système européen et atlantique structuré : la majorité de la classe politique ouest-allemande et la plupart des partenaires de l'Allemagne préfèrent cette solution à un statut de neutralité, par exemple, qui rappellerait trop l'Europe d'avant Yalta. La formule mise en avant par Hans-Dietrich Genscher, qui propose deux volets, l'appartenance politique de l'Allemagne à l'OTAN mais non l'intrégration militaire de l'Allemagne orientale, constitue un compromis susceptible d'emporter l'adhésion des Européens comme celle des Grands. Elle ménage en effet les intérêts des uns et des autres puisqu'elle n'affaiblit ni ne renforce l'OTAN tout en maintenant l'Allemagne dans un réseau d'engagements. Elle permet aussi de gagner du temps en attendant que les membres de l'Alliance atlantique et du pacte de Varsovie s'accordent - ou non - pour transformer le système européen et atlantique. Cependant, cette formule intermédiaire est nécessairement intérimaire car elle pose à l'Allemagne autant de problèmes qu'elle en résoud. Des troupes allemandes seront-elles stationnées en Allemagne orientale ? Y aura-t-il à Berlin un ministre de la 
Défense dont les compétences ne s'étendront que du Rhin à l'Elbe ? L'Allemagne n'aura-t-elle pas le droit de se défendre à ses frontières ? Pour contourner ces difficultés, le SPD met en avant la nécessité de transformer les alliances en un système de sécurité collective - dont il se fait d'ailleurs l'avocat depuis quelque temps déjà et dont le libéral ministre des Affaires étrangères, Hans-Dietrich Genscher, ne repousse pas, à terme, l'idée. Un tel système pourra-t-il cependant éviter les écueils des précédents et résoudra-t-on notamment la question de qui décide quoi ?

Si la chute des régimes communistes et la réunification des Allemagnes apportent à l'Allemagne et à l'Europe plus de souveraineté mais non nécessairement plus de sécurité, elles entraînent aussi Bonn et Berlin-Est et plus tard Berlin - dans des turbulences économiques et politiques. Certes, à terme, on peut penser avec Helmut Haussmann, le ministre ouestallemand de l'Economie, que la réunification des Allemagnes, la constitution d'un seul espace économique et monétaire et la nécessité de reconstruire rapidement l'Allemagne orientale accéléreront la croissance allemande : on pourra parler d'un nouveau miracle économique allemand à l'horizon 2000 dont l'Europe occidentale profitera peu ou prou (on estime à $0,5 \%$ son gain de croissance). Entre-temps, l'introduction du mark allemand en Allemagne orientale et la prise en charge par Bonn ou Francfort du système social est-allemand affaibliront la monnaie allemande - au moment même où des sommes considérables devront être investies dans la partie orientale de l'Allemagne. Les hausses de taux d'intérêt qui s'ensuivront perturberont le Système monétaire européen. Pour toutes ces raisons, l'unification économique et monétaire des Douze souffrira vraisemblablement de retards. La constitution d'un espace économique et monétaire n'aura cependant pas des effets immédiats sur l'économie est-allemande : il faudra près de dix ans et 800 milliards de marks allemands pour amener la RDA à un niveau quelque peu semblable à celui de la RFA ! L'introduction du mark allemand en RDA jouera certes le rôle d'un déclencheur psychologique, les Allemands de l'Est se mettront à travailler de meilleur cour pour une monnaie solide. Pourtant, on peut s'attendre à ce que les départs d'Est en Ouest continuent longtemps encore - même à un rythme plus lent.

Sur le plan politique, la constitution d'une grande Allemagne ne se fera pas sans débats ni sans heurts. Avec l'ouverture des frontières le 9 novembre 1989 et l'annonce du plan Kohl, le 28 novembre, avec surtout la double tenue d'élections, est-allemandes le 18 mars 1990 et ouest-allemandes le 2 décembre, qui haussent les enchères, des débats s'entrecroisent, dans chacune des Allemagnes et de l'une à l'autre. Chacune pèse désormais dans la vie publique de l'autre. Les partis politiques de République fédérale mènent en RDA une double campagne, celle qui conduira aux élections du 18 mars et celles du 2 décembre car, le soir du 18, il y aura deux vainqueurs, le parti ou la coalition majoritaire à l'Est et le parti qui, à l'Ouest, l'aura soutenu, conseillé et financé. Mais la République démocratique pèse aussi en République fédérale puisqu'il n'y a plus désormais à Bonn qu'un débat aux multiples facettes : le discours sur l'unité, ses modalités et ses coûts. Au-delà des échéances électorales, chacun prend ses marques, avec pour enjeu la recomposition du paysage politique de l'Allemagne unifiée. Avec l'adjonction des anciens bastions orientaux, la social-démocratie espère devenir le plus grand parti d'Allemagne - alors que, jusqu'à une date 
récente, elle ignora le langage de l'unité. A droite, le chancelier Kohl, artisan de l'unité, doit ménager ses différents électorats en promettant à la fois aux Allemands de l'Est de leur garantir un minimum social et à ceux de l'Ouest de ne pas augmenter leurs impôts ; il risque sinon de perdre une partie de sa clientèle libérale et conservatrice en RFA et, en Allemagne orientale, de s'aliéner les voix de ceux qui craignent le bradage de leur pays. Même démunis, les Allemands de l'Est veulent en effet se faire entendre et modeler la future Allemagne.

16 millions d'Allemands de l'Est modifieront nécessairement la culture politique allemande ; certains n'ont jamais pratiqué la démocratie, il leur faudra l'apprendre. De la gauche à la droite, du Nouveau forum aux extrémistes de droite, futurs Republikaner, les Allemands de l'Est seront tout autant divisés que l'est la société ouest-allemande. Avec une telle diversité géographique, politique, culturelle, avec des institutions qui ne renforceront certainement pas le pouvoir central, avec peut-être enfin l'impossibilité pour tout parti de parvenir seul au pouvoir, l'Allemagne ne sera pas plus facile à gouverner que la République fédérale ne l'est, d'autant qu'elle devra compter avec une petite minorité, d'intellectuels notamment, qui n'accepteront pas l'Etat unitaire. Il est à parier que les futurs chanceliers auront autant de mal qu'Helmut Kohl dans les années 80 à concilier les intérêts divers et, partant, à parler d'une seule voix ferme sur la scène internationale : l'Allemagne parviendra-t-elle alors à tenir tous ses engagements vis-à-vis de l'étranger ? 